\title{
Maladaptive personality traits, psychological morbidity and coping strategies in chronic central serous chorioretinopathy
}

\author{
Femke M. van Haalen, ${ }^{1, \dagger}$ Elon H.C. van Dijk, ${ }^{2, \dagger}$ (D) Cornelie D. Andela, ${ }^{1}$ Greet Dijkman, ${ }^{2}$ \\ Nienke R. Biermasz, ${ }^{1}$ Alberto M. Pereira ${ }^{1}$ and Camiel J.F. Boon ${ }^{2,3}$ (D) \\ ${ }^{1}$ Department of Medicine, Division of Endocrinology and Center for Endocrine Tumors, Leiden University Medical Center, Leiden, \\ the Netherlands \\ ${ }^{2}$ Department of Ophthalmology, Leiden University Medical Center, Leiden, the Netherlands \\ ${ }^{3}$ Department of Ophthalmology, Amsterdam University Medical Center, University of Amsterdam, Amsterdam, the Netherlands
}

\begin{abstract}
.
Purpose: 'Type A' behavioural characteristics and psychosocial stress have traditionally been associated with chronic central serous chorioretinopathy (cCSC). However, a characteristical personality profile could not be identified in these patients and the presumed association with stress is subject to controversy, due to a lack of convincing studies using validated measuring instruments. In this study, we aimed to assess maladaptive personality traits, psychological morbidity and coping strategies in patients with cCSC, in order to identify potentially modifiable psychosocial aspects which could be used in support to current standard treatment.

Methods: A cross-sectional study in a cohort of 86 patients with cCSC using validated questionnaires. Findings were compared to both Dutch population reference data and reference data from patients treated for Cushing's disease.

Results: Maladaptive personality traits were not more prevalent in patients with cCSC than in the general population, and psychological morbidity was not increased. Patients with cCSC were shown to make more use of passive coping, active coping and seeking social support. Interestingly, personality, psychological morbidity and coping characteristics of patients with cCSC were more comparable to features of patients treated for Cushing's disease than to population-based data.

Conclusion: Maladaptive personality traits such as type $\mathrm{A}$ behavioural characteristics are not more prevalent in patients with cCSC. Patients with cCSC make more use of certain coping strategies, which could be addressed by psychosocial care to improve self-management. Further research is needed establish whether the course of disease can be improved by altering coping and reducing 'stress'.
\end{abstract}

Key words: central serous chorioretinopathy-coping-cross-sectional study-irritability personality - stress

Acta Ophthalmol.

(c) 2018 Acta Ophthalmologica Scandinavica Foundation. Published by John Wiley \& Sons Ltd

doi: 10.1111 aos. 13952

\section{Introduction}

Central serous chorioretinopathy (CSC) is a specific and relatively common chorioretinal disease in which choroidal congestion, thickening and hyperpermeability damage the retinal pigment epithelium and subsequently induce serous subretinal fluid accumulation and detachment of the neuroretina.

The pathogenesis of CSC is currently unclear, but many studies indicate a pathophysiological association with stress pathways, due to the relation with both exogenous and endogenous corticosteroid excess, as well as overactivity of the hypothalamus-pituitary-adrenal (HPA) axis (Bouzas et al. 2002; Carvalho-Recchia et al. 2002; Jonas \& Kamppeter 2005; Wang et al. 2008; Gemenetzi et al. 2010; Liew et al. 2013; Nicholson et al. 2013; van Dijk et al. 2016; van Haalen et al. 2018). Both acute and chronic psychosocial stress have been suggested to predispose to CSC (Conrad et al. 2000; Spahn et al. 2004). It has also been suggested that people with a type A behaviour pattern have an increased risk to develop CSC (Jenkins et al. 1967; Yannuzzi 1987; Baraki et al. 2010; Chatziralli et al. 2017). The term 'type A behaviour' was introduced by Friedman \& Rosenman (1959) and was characterized as follows: an intense, sustained drive to achieve self-selected but usually poorly defined goals, 
profound inclination and eagerness to compete, persistent desire for recognition and advancement, continuous involvement in multiple and diverse functions constantly subject to deadlines, habitual propensity to accelerate the rate of execution of many physical and mental functions, and extraordinary mental and physical alertness. The concept of personality types has been studied in medical psychology as a predictor of the cause, course and quality of life of somatic diseases such as cancer, rheumatic diseases and coronary artery disease (Dalton et al. 2002; Hausteiner et al. 2010; Donisan et al. 2017). It has been hypothesized that type A behaviour might be linked to CSC by increased levels of circulating catecholamines and corticosteroids, since these hormone levels are found to be higher in people with type A behavioural characteristics compared to those with type B behavioural characteristics (more relaxed and less hurried) (Friedman et al. 1960, 1975; Rosenman et al. 1976; Williams et al. 1982). A recent meta-analysis indeed concluded that patients with CSC demonstrated significantly more type A behavioural characteristics than healthy controls (odds ratio $(\mathrm{OR})=2.53$; confidence interval $(\mathrm{CI})$ 1.08-5.96) (Liu et al. 2016). Despite this proposed association between CSC and type A behavioural characteristics (Yannuzzi 1987), a typical CSC personality profile could not be identified in previous studies. Only type A behavioural characteristics have been previously observed (Bahrke et al. 2000).

It is well known that personality affects coping behaviour (Friedman et al. 2006). Coping behaviour encompasses the way people react on a behavioural, cognitive and emotional level to situations that require adjustments in dealing with possible adverse events (Schreurs et al. 1993), which has an effect on the amount of stress experienced (Ersan et al. 2017). Specific coping styles (e.g. emotion-oriented coping) have even been reported to have an effect on disease severity, for example in multiple sclerosis (Brands et al. 2017). For CSC, several mostly small-sized studies have reported an association between severe psychosocial stressful events and the onset of disease, with one study describing this association especially in patients with poor coping mechanisms (Conrad et al. 2000; Spahn et al. 2004; Lahousen et al. 2016). However, coping behaviour may be a valuable starting point for psycho-education or selfmanagement training in order to improve quality of life. Also psychological morbidity such as apathy or irritability may be a potential point of engagement for self-management programmes. To date, these psychological factors have not been evaluated in CSC patients.

To the best of our knowledge, no previous systematic studies have been published assessing personality traits in patients with CSC, and there are no studies in a large cohort of patients with CSC that have systematically evaluated coping strategies using a specific coping-oriented validated questionnaire. Since CSC seems to be related to stress (Bouzas et al. 2002; Carvalho-Recchia et al. 2002; Jonas \& Kamppeter 2005; Wang et al. 2008; Gemenetzi et al. 2010; Liew et al. 2013; Nicholson et al. 2013; van Dijk et al. 2016; van Haalen et al. 2018), a detailed assessment of potential associations with personality traits, psychological morbidity and coping mechanisms is essential to identify potentially psychosocial aspects that could be modifiable with self-management programmes.

The primary aim of this study was to assess maladaptive personality traits (i.e. traits related to type $\mathrm{A}$ behavioural pattern), in patients with cCSC. For this purpose, we compared personality traits of patients with cCSC to personality traits of Dutch population reference data, but also to personality traits of patients treated for Cushing's disease (since these patients were exposed to excessive HPA - axis activity). In addition, this study aimed to assess psychological morbidity (i.e. apathy and irritability) and coping strategies in patients with cCSC by comparing these patients with the same reference groups. Finally, we aimed to assess the association between personality and coping in patients with cCSC. Since previous studies have pointed towards a higher prevalence of type A behavioural characteristics in patients with cCSC and considering the above-mentioned definition of type A behaviour (Friedman \& Rosenman 1959), we assessed whether patients with cCSC report more stimulus seeking, callousness, rejection, conduct problems and narcissism. Furthermore, considering the recently described hyperactivity of the HPA axis in patients with cCSC (van Haalen et al. 2018) and the previously described maladaptive personality traits in patients exposed to hypercortisolism (i.e. Cushing's disease) (Tiemensma et al. 2010a,b), we hypothesized that patients with CSC would report more maladaptive personality traits, more psychological morbidity (i.e. apathy, irritability) and less effective coping strategies compared to reference data from the general population. In accordance with previous literature in patients with other chronic diseases (Schouws et al. 2015; Vollmann et al. 2016; Keramat Kar et al. 2017; Yadav et al. 2017; You et al. 2018), we hypothesized that more maladaptive personality traits are associated with less effective coping strategies in patients with cCSC.

\section{Subjects and Methods}

\section{Study design}

We conducted a cross-sectional study in a cohort of patients with cCSC. Patients were asked to complete a set of validated questionnaires on personality traits, psychological morbidity (i.e. apathy and irritability) and coping strategies at home, using an online survey. In addition, a clinical evaluation took place during a single visit to the outpatient clinic of the Division of Endocrinology of the Leiden University Medical Center.

\section{Study population}

Eighty-six consecutive adult patients with cCSC, who were followed at the Department of Ophthalmology of Leiden University Medical Center, a tertiary referral center for CSC, were invited to complete the questionnaires. The cCSC diagnosis had been confirmed by fundoscopy, digital colour fundus photography (Topcon Corp., Tokyo, Japan), fundus autofluorescence (Spectralis Heidelberg retinal angiography (HRA) + optical coherence tomography (OCT); Heidelberg Engineering, Heidelberg, Germany), spectral-domain OCT (Spectralis HRA + OCT), fluorescein angiography (Spectralis HRA + OCT) and 
indocyanine green angiography (Spectralis HRA + OCT), according to current standards (Wang et al. 2008; Gemenetzi et al. 2010; Yannuzzi 2010; Liew et al. 2013; Nicholson et al. 2013; Daruich et al. 2015). On multimodal imaging, the following characteristics had to be present within the past 2 years: serous subretinal fluid on OCT, $\geq 1$ area of multifocal diffuse leakage or irregular retinal pigment epithelium window defects on fluorescein angiography, and corresponding hyperfluorescence on indocyanine green angiography. Patients were divided into active or nonactive cCSC at the moment of evaluation, defined by the presence of subretinal fluid. We excluded patients diagnosed with acute CSC, defined by either a focal leakage spot or a smoke stack pattern on fluorescein angiography (Wang et al. 2008; Gemenetzi et al. 2010; Yannuzzi 2010; Liew et al. 2013; Nicholson et al. 2013; Daruich et al. 2015), as well as patients in whom evidence of other retinal diagnoses was detected. The patients also participated in a study on endocrinological phenotyping focussed on the HPA axis (data presented elsewhere) (van Haalen et al. 2018), for which other exclusion criteria were the use of corticosteroids or sleep medication prior to the development or during the time-course of cCSC, excessive alcohol intake ( $>21$ units/week), either night shift work or travelling from another time zone in the 6 weeks prior to evaluation. Endocrinological evaluation of the patients included a detailed medical history and complete physical examination and was performed by two endocrinologists. After reassessment of the retinal imaging by two independent ophthalmologists, five patients were considered to have less typical cCSC findings on imaging. Written informed consent was obtained from all participants, and approval of the institutional review board and the ethics committee was obtained (NL50816.058.14). Research was conducted following the tenets of the Declaration of Helsinki.

\section{Questionnaires}

Dimensional assessment of personality pathology short form

This questionnaire consists of 136 items assessing personality, which are subdivided into 18 subscales: submissiveness, cognitive distortion, identity problems, affective lability, stimulus seeking, compulsivity, restricted expression, callousness, oppositionality, intimacy problems, rejection, anxiousness, conduct problems, suspiciousness, social avoidance, narcissism, insecure attachment and self-harm (van Kampen et al. 2008; de Beurs et al. 2009). The maximal scores for each subscale differ from 30 to 40, and higher scores indicate more pronounced maladaptive personality traits. No formal cut-off scores for these subscales exist (van Kampen et al. 2008; de Beurs et al. 2009). We hypothesized that if type A behavioural characteristics would be more prevalent in patients with cCSC, these patients would report more stimulus seeking, callousness, rejection, conduct problems and narcissism.

\section{Apathy Scale}

The Apathy Scale (AS) of Starkstein was used to assess apathy (Starkstein et al. 2001). The scale consists of 14 questions on a four-point scale, measuring different features of apathy in the two previous weeks. Total scores in a range from 0 to 42 points are calculated, with higher scores indicating greater apathy. A total score of 14 points or more defines apathy (Chatterjee et al. 2005).

\section{Irritability Scale}

Irritability was assessed by the Irritability Scale (IS) (Chatterjee et al. 2005). This scale consists of 14 items on a four-point scale, assessing different features of irritability in the two previous weeks. Total scores range from 0 to 42 points, with higher scores indicating greater irritability. A total score of 14 points or more defines irritability.

\section{Utrecht Coping Scale}

The Utrecht Coping Scale (UCS) is an established Dutch coping list with welldocumented validity and reliability (Hopman-Rock et al. 1997). It contains 47 statements where one indicates whether he/she finds these applicable to him- or herself. This scale assesses the way a person acts to minimize the impact of stressful events, with seven subscales that represent different coping styles. These subscales include active coping (i.e. immediate action in case of problems, considering problems as a challenge, keeping calm, goaloriented problem-solving), distractionseeking, avoidance, seeking social support, passive coping (i.e. isolation, worrying about the past, using soothing resources, fleeing in fantasies), expression of emotions, and positive reframing (i.e. optimism, trying to reconsider things in a positive light). The different items have a four-point scale ranging from 1 (seldom or never) to 4 (very often). Item scores on each subscale are summed to create a total score, with scores of 4 or 5 indicating high use of that specific coping style (Schreurs et al. 1984). Data from an aselect sample of the Dutch railway workers (1493 men, aged between 19 and 65 years) were used as reference data. A cohort of 42 Cushing's disease patients (six men and 36 women) with a mean age of $54( \pm 12)$ years was used for comparison (Tiemensma et al. 2011).

\section{Reference data}

Outcomes of the questionnaires were compared to reference of a random sample of the Dutch population and reference data from patients treated for Cushing's disease as reported previously by Tiemensma et al. (2010a,b, 2011). For comparison of dimensional assessment of personality pathology short form (DAPPsf) outcomes, reference data from the publisher of this questionnaire were available (Dirk van Kampen 2009). The sample used for obtaining these data consisted of 58 men aged 15-34 years, 94 men aged 35-54 years, 146 women aged 15-34 years and 172 women aged 3554 years. The sample of patients treated for Cushing's disease used for comparison consisted of eight men and 43 women with a mean age of 53 $( \pm 13)$ years (Tiemensma et al. 2010a, b). Concerning the AS and IS, reference data were derived from the healthy control population described by Tiemensma et al. $(2010 \mathrm{a}, \mathrm{b})$ consisting of 35 men and 33 women with a mean age of $59( \pm 11)$ years. No maleonly reference data were available. The same cohort of patients with Cushing's disease was used for the comparison of the AS and IS scores (Tiemensma et al. 2010a,b). Data from a random sample of the Dutch railway workers (1493 men, aged between 19 and 65 years) 
were used as reference data for the comparison of UCS outcomes. Moreover, the cohort of 42 patients treated for Cushing's disease (six men and 36 women) with a mean age of $54( \pm 12)$ years was used for comparison (Tiemensma et al. 2011).

\section{Statistical analysis}

Data were presented as mean and standard deviation (SD), unless mentioned otherwise. The primary analyses comprised the comparison of questionnaire outcomes between patients with cCSC and reference data from the general population. Secondary analyses comprised the comparison between patients with cCSC and patients treated for Cushing's disease. Groups were compared using pooled $t$-tests. The level of significance was set at $\mathrm{p} \leq 0.01$ in order to correct for multiple testing. Normality of data was tested using the Shapiro-Wilk test. Correlations between personality and coping were assessed using Pearson's correlation in case of normally distributed data, and data with a nonnormal distribution were correlated using Spearman correlation. Only moderate-to-strong correlations (correlation coefficient of $>0.5$ ) were described.

A post hoc sensitivity analysis excluding the five less typical cCSC patients was performed. Data were analysed using sPSS Statistics (version 23; IBM Corp., Armonk, New York, USA).

\section{Results}

\section{Baseline characteristics}

A total of 86 patients with cCSC (77 males [90\%]) with a mean age of 48.7 years (range, 24-77 years) were included. In all patients, subretinal fluid had been present $<2$ years ago. In 58 patients with cCSC $(67 \%)$, subretinal fluid was present at the moment of evaluation, indicating active cCSC. The mean duration from first cCSC diagnosis at an ophthalmologist to inclusion in our study was 3.9 years (range, 0.2-37.1 years). A history of hypertension was reported by 23 patients $(27 \%)$, dyslipidaemia by 18 patients $(21 \%)$ and psychiatric disorders by 16 patients $(19 \%)$ (Table 1$)$. Apart from being slightly overweight (mean body mass index $26.2 \mathrm{~kg} / \mathrm{m}^{2}$ ), patients appeared to be healthy on physical examination, with a mean blood pressure within the normal range. None of the patients fulfilled the criteria for Cushing's syndrome.

\section{Personality traits}

Dimensional assessment of personality pathology short form

The DAPPsf was completed by 81 patients with cCSC (94\%). Compared to reference data from the general population, patients with cCSC reported only more intimacy problems $(\mathrm{p}<0.01)$, but less submissiveness $(\mathrm{p}<0.01)$, less cognitive distortion ( $\mathrm{p}$ $<0.01)$, less affective lability ( $\mathrm{p}<$ 0.01 ), less stimulus seeking ( $p<0.01$ ), less compulsivity $(\mathrm{p}<0.01)$, less oppositionality $(\mathrm{p}<0.01)$, less anxiousness $(\mathrm{p}<0.01), \quad$ less suspiciousness $(p<0.01)$, less social avoidance $(\mathrm{p}<0.01)$, less narcissism $(\mathrm{p}<0.01)$ and less insecure attachment $(p<0.01)$ (Table 2 and Fig. 1). Interestingly, there was no increased prevalence of type A behavioural characteristics in patients with cCSC (i.e. no more stimulus seeking, callousness, rejection, conduct problems and narcissism).

Compared to patients treated for Cushing's disease, patients with cCSC reported more conduct problems $(\mathrm{p}<0.01)$, but less affective lability $(\mathrm{p}<0.01)$, less cognitive distortion $(\mathrm{p}<0.01)$ and less oppositionality $(\mathrm{p}<0.01)$. For the remaining personality traits, no large difference was observed between patients with cCSC and patients treated for Cushing's disease.

Compared to patients with active cCSC $(n=54)$, patients with inactive disease $(n=27)$ reported more affective lability, submissiveness and social avoidance $(\mathrm{p}<0.01, \quad \mathrm{p}<0.01$ and $\mathrm{p}<0.01$, respectively).

\section{Psychological morbidity}

The AS was completed by 83 patients with cCSC (97\%) (Table 3). The mean score of patients with cCSC was 12.2 (range, 3-26). Clinically relevant apathy (a score of $\geq 14$ ) was present in $34.9 \%$ of the patients with cCSC. No differences in reported apathy were found between patients with cCSC and the reference data from the general population, and the apathy score was lower than scores of patients treated for Cushing's disease ( $p=0.03$ and $p=0.01$, respectively). Although no significant differences were found, the scores of patients with cCSC were in between the scores of the reference data from the general population and the reference data from patients treated for Cushing's disease (Fig. 2). No difference was observed in total scores between patients with active cCSC and patients with inactive disease $(\mathrm{p}=0.26)$.

The IS was also completed by 83 patients with cCSC (97\%) (Table 3). Mean patient score was 9.8 (range, 0 26). Clinically relevant irritability (a score of $\geq 14$ ) was present in $29.3 \%$ of patients with cCSC. No differences in

Table 1. Clinical characteristics of chronic central serous chorioretinopathy (cCSC) patients.

\begin{tabular}{lc}
\hline & $\begin{array}{l}\text { cCSC patients } \\
n=86\end{array}$ \\
\hline Mean age, years (SD) & $48.7(10.8)$ \\
Sex, male/female & $77 / 9$ \\
Duration of cCSC disease, years (range) & $3.9(0.2-37.1)$ \\
History of hypertension, $n(\%)$ & $23(26.7 \%)$ \\
History of diabetes mellitus, $n(\%)$ & $6(7.0 \%)$ \\
History of dyslipidaemia, $n(\%)$ & $18(20.9 \%)$ \\
History of psychiatric disorders $\%, n(\%)$ & $16(18.6 \%)$ \\
History of thromboembolic events, $n(\%)$ & $0(0 \%)$ \\
History of cardiac events ${ }^{\dagger}, n(\%)$ & $5(5.9 \%)$ \\
History of sexual disorders ${ }^{*}, n(\%)$ & $19(22.1 \%)$ \\
\hline
\end{tabular}

$\mathrm{SD}=$ standard deviation.

* Consisting of depression, anxiety or panic disorder, posttraumatic stress disorder, burnout, alcohol abuse and schizophrenia.

${ }^{\dagger}$ Consisting of myocardial infarction, endocarditis and atrial fibrillation.

Consisting of impotence, hirsutism, menstrual cycle disorders and loss of libido. 
Table 2. Personality traits in chronic central serous chorioretinopathy (cCSC).

\begin{tabular}{|c|c|c|c|c|c|}
\hline DAPPsf & cCSC patients $(n=81)$ & Reference data $(n=475)$ & p-Value & Cushing's disease patients $(n=51)$ & p-Value \\
\hline Submissiveness & $16.2(6.6)$ & $19.7(6.3)$ & $<0.01$ & $19.0(7.7)$ & 0.03 \\
\hline Cognitive distortion & $9.0(4.3)$ & $12.1(5.4)$ & $<0.01$ & $11.5(5.6)$ & $<0.01$ \\
\hline Identity problems & $10.6(4.7)$ & $12.2(5.6)$ & 0.02 & $13.0(6.6)$ & 0.01 \\
\hline Affective lability & $16.5(6.9)$ & $21.0(7.3)$ & $<0.01$ & $21.7(7.8)$ & $<0.01$ \\
\hline Stimulus seeking & $15.2(5.4)$ & $18.0(5.8)$ & $<0.01$ & $16.4(4.8)$ & 0.19 \\
\hline Compulsivity & $21.9(7.1)$ & $24.2(6.5)$ & $<0.01$ & $23.8(6.6)$ & 0.12 \\
\hline Restricted expression & $20.9(5.9)$ & $21.3(6.5)$ & 0.65 & $21.2(7.3)$ & 0.80 \\
\hline Callousness & $17.2(5.2)$ & $18.8(5.4)$ & $<0.01$ & $16.1(4.5)$ & 0.23 \\
\hline Oppositionality & $19.0(7.0)$ & $23.1(7.2)$ & $<0.01$ & $22.9(8.8)$ & $<0.01$ \\
\hline Intimacy problems & $20.9(5.9)$ & $16.9(5.7)$ & $<0.01$ & $18.8(6.4)$ & 0.06 \\
\hline Rejection & $19.3(6.7)$ & $20.1(5.7)$ & 0.28 & $17.2(5.7)$ & 0.06 \\
\hline Anxiousness & $12.8(6.2)$ & $17.8(5.7)$ & $<0.01$ & $15.3(6.2)$ & 0.03 \\
\hline Conduct problems & $10.8(4.8)$ & $11.5(4.4)$ & 0.21 & $9.0(1.8)$ & $<0.01$ \\
\hline Suspiciousness & $12.7(5.4)$ & $15.0(5.9)$ & $<0.01$ & $12.6(5.9)$ & 0.96 \\
\hline Social avoidance & $11.0(5.2)$ & $13.8(5.5)$ & $<0.01$ & $12.3(6.3)$ & 0.20 \\
\hline Narcissism & $15.7(5.7)$ & $18.7(6.2)$ & $<0.01$ & $15.0(5.5)$ & 0.47 \\
\hline Insecure attachment & $10.9(4.9)$ & $13.7(5.6)$ & $<0.01$ & $13.3(6.6)$ & 0.02 \\
\hline Self-harm & $7.0(2.7)$ & $8.0(4.2)$ & 0.05 & $7.3(2.9)$ & 0.58 \\
\hline
\end{tabular}

Data are presented as mean (SD).

$\mathrm{cCSC}=$ chronic central serous chorioretinopathy; DAPPsf $=$ Dimensional assessment of personality pathology short form; $\mathrm{SD}=$ standard deviation.

reported irritability were observed between patients with cCSC and the reference data from the general population $(\mathrm{p}=0.79)$, nor to reference data from patients treated for Cushing's disease $(p=0.15)$. Although no statistically significant differences were found, the scores of patients with cCSC were in between the scores of the reference data from the general population and the reference data from patients treated for Cushing's disease, which was in line with the outcome of the assessment of apathy (Fig. 2). Total scores did not differ between patients with active cCSC and patients with inactive $\mathrm{cCSC}(\mathrm{p}=0.36)$.

\section{Coping strategies}

Eighty-three patients with cCSC (97\%) completed the UCS (Table 4). Compared to the reference data, patients with cCSC reported to use more passive coping strategies and to seek more social support $(\mathrm{p}<0.01$ and $\mathrm{p}<0.01$, respectively). Because the reference data were male only, data from male patients with cCSC between 19 and 65 years of age $(n=67)$ were compared separately. This category of patients with cCSC made more use of active coping compared to the reference population $(p<0.01)$, in addition to the aforementioned seeking social support $(\mathrm{p}<0.01)$ and passive coping $(\mathrm{p}<0.01)$. Patients with inactive disease $(n=27)$ made more use of avoiding compared to cCSC patients with active disease $(n=56, \mathrm{p}<0.01)$. No differences in coping strategies were observed between patients with cCSC and patients treated for Cushing's disease.

\section{Correlation between personality and coping strategies in cCSC}

Moderate-to-strong correlations were found between several maladaptive personality traits and passive coping. More affective lability $(\mathrm{p}<0.01$, $\left.R^{2}=0.759\right), \quad$ cognitive distortion (p $\left.<0.01, R^{2}=0.656\right)$, identity problems $\left(\mathrm{p}<0.01, R^{2}=0.675\right)$, insecure attachment $\quad\left(\mathrm{p}<0.01, \quad R^{2}=0.558\right)$, oppositionality $\left(\mathrm{p}<0.01, R^{2}=0.522\right)$, social avoidance $\left(\mathrm{p}<0.01, R^{2}=0.515\right)$ and anxiousness $\left(\mathrm{p}<0.01, R^{2}=0.711\right)$ correlated with using more passive coping.

Post hoc analysis without patients with less typical cCSC

Analyses performed without the five patients with atypical cCSC revealed only a few minor differences. These results are shown in Appendix S1.

\section{Discussion}

In this study, personality traits, psychological morbidity and coping strategies were systematically assessed in a cohort of patients with cCSC. We did not find a higher prevalence of maladaptive personality traits such as type A behavioural characteristics in cCSC as compared to the general population, which is in contrast to what has been suggested previously (Yannuzzi 1987; Baraki et al. 2010; Liu et al. 2016; Chatziralli et al. 2017). On the level of conduct, patients did not report more psychological morbidity in the form of apathy or irritability. Patients with cCSC made more use of certain coping strategies (e.g. seeking social support, passive coping, and in males also active coping) compared to the general population.

In contrast to earlier studies suggesting more type A behavioural characteristics (i.e. persistent desire for recognition and advancement, and habitual propensity to accelerate the rate of execution of many physical and mental functions) in these patients (Yannuzzi 1987; Baraki et al. 2010; Liu et al. 2016; Chatziralli et al. 2017), we did not find any evidence to support this. Critical evaluation of the available literature revealed that type A behavioural characteristics were mainly assessed using behavioural outcome measures (i.e. Jenkins activity survey) in previous studies (Yannuzzi 1987; Baraki et al. 2010; Liu et al. 2016; Chatziralli et al. 2017), while this inventory has been shown not to correlate with personality characteristics and psychopathology (Wadden et al. 1983). In another study, type A 


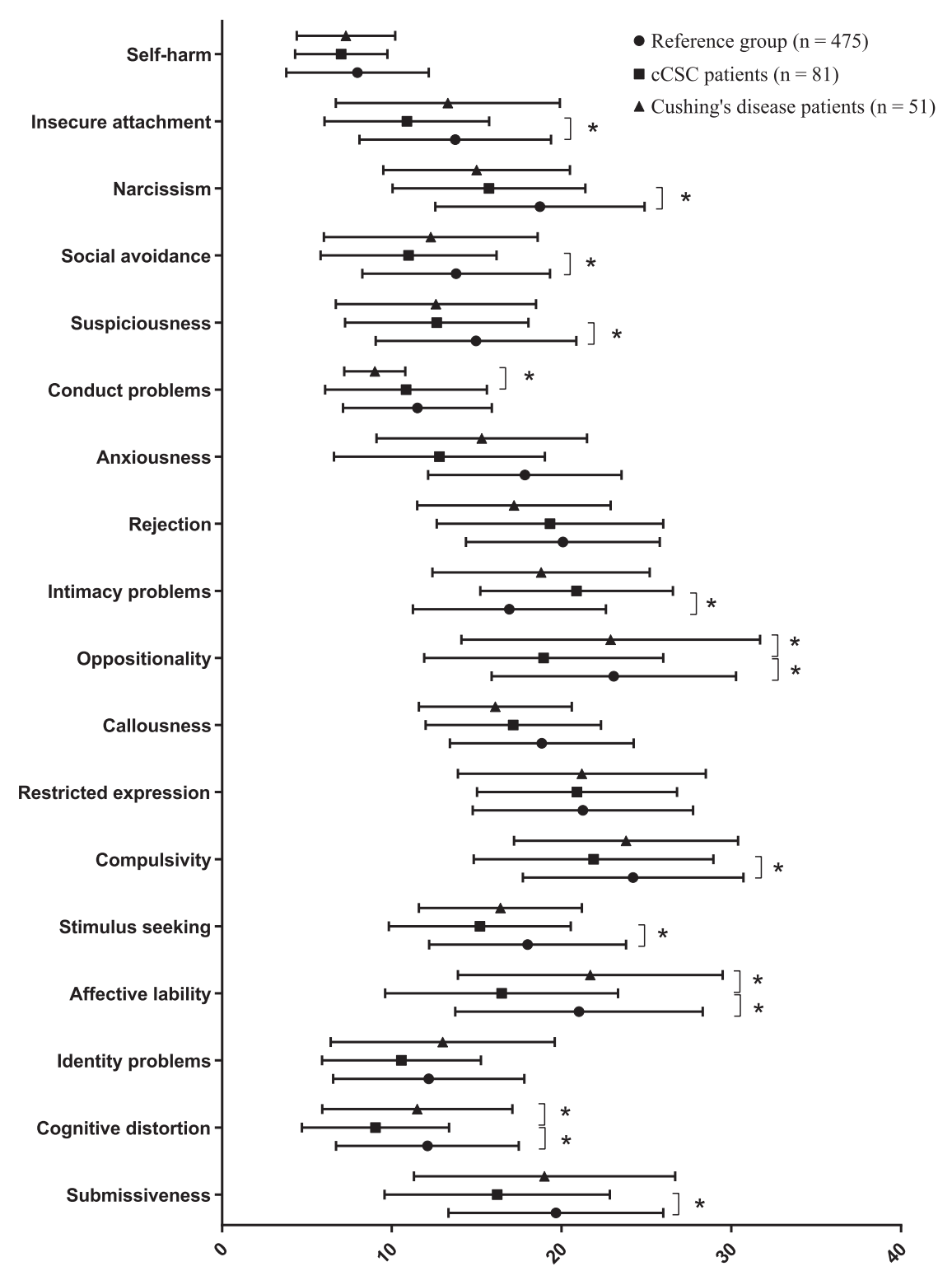

Fig. 1. DAPPsf personality traits in patients with cCSC. Data are presented as mean and SD. $\mathrm{cCSC}=$ chronic central serous chorioretinopathy; DAPPsf $=$ Dimensional assessment of personality pathology short form; $\mathrm{SD}=$ standard deviation; $*=$ statistically significant (defined as p-value $<0.01)$.

Table 3. Apathy and irritability in patients with cCSC.

\begin{tabular}{llllll}
\hline & & & & \\
& $\begin{array}{l}\text { Clshing's } \\
\text { disease patients } \\
(n=83)\end{array}$ & $\begin{array}{l}\text { Reference } \\
\text { data }(n=68)\end{array}$ & p-Value & p-Value \\
\hline Apathy & $12.2(5.0)$ & $10.5(4.8)$ & 0.03 & $14.8(6.5)$ & 0.01 \\
Irritability & $9.8(6.2)$ & $9.5(5.7)$ & 0.79 & $11.5(7.7)$ & 0.15 \\
\hline
\end{tabular}

Data are presented as mean (SD).

$\mathrm{cCSC}=$ chronic central serous chorioretinopathy; $\mathrm{SD}=$ standard deviation.

behavioural characteristics were not strictly defined, as the term itself may have appeared in the medical charts or patients were included as being type A based on a description of patients by themselves, family members or physicians as being 'tense', 'high strung' or 'highly ambitious' (Breukink et al. 2017). The conclusion of a recent meta-analysis suggesting more type A behavioural characteristics in patients with cCSC was based on these small studies lacking a type A phenotyping protocol, making the conclusion less reliable (Liu et al. 2016).

The fact that patients with cCSC seem to report more intimacy problems was interpreted as a chance finding, since all the other traits point in the opposite direction, with generally less maladaptive personality traits in patients with cCSC compared to the general population. Interestingly, the personality profile of patients with cCSC in our cohort tended towards more similarities with the profile of patients treated for Cushing's disease than to the general population, since 14 out of the 18 DAPPsf subscales outcomes of patients with cCSC were comparable with outcomes of patients treated for Cushing's disease, where only eight out of 18 were comparable with the general population (Fig. 1, with lines resembling patients with cCSC in between the lines corresponding with reference data of the general population and patients treated for Cushing's disease). However, these findings were not statistically significant.

Cushing's disease is a rare condition which is characterized by exposure to excessive cortisol levels. Therefore, these patients can be regarded as a human model to study the effects of cortisol excess on personality and behaviour. Maladaptive personality traits and psychological morbidity, such as somatic arousal, negative affect, irritability and apathy, have well been documented in patients with Cushing's disease (Tiemensma et al. 2010a,b). Patients with cCSC showed less affective lability, cognitive distortion and oppositionality compared to patients treated for Cushing's disease, whereas they reported more conduct problems, although the significance of this difference was omitted in the post hoc analysis excluding patients with less typical cCSC. Apathy and irritability scores of patients with cCSC in our cohort were lower, yet not statistically significant compared to scores of patients treated for Cushing's disease. We have recently demonstrated that patients with cCSC have an activated HPA axis in the presence of high normal serum levels of cortisol (van Haalen et al. 2018). In line with this biochemical resemblance of an 


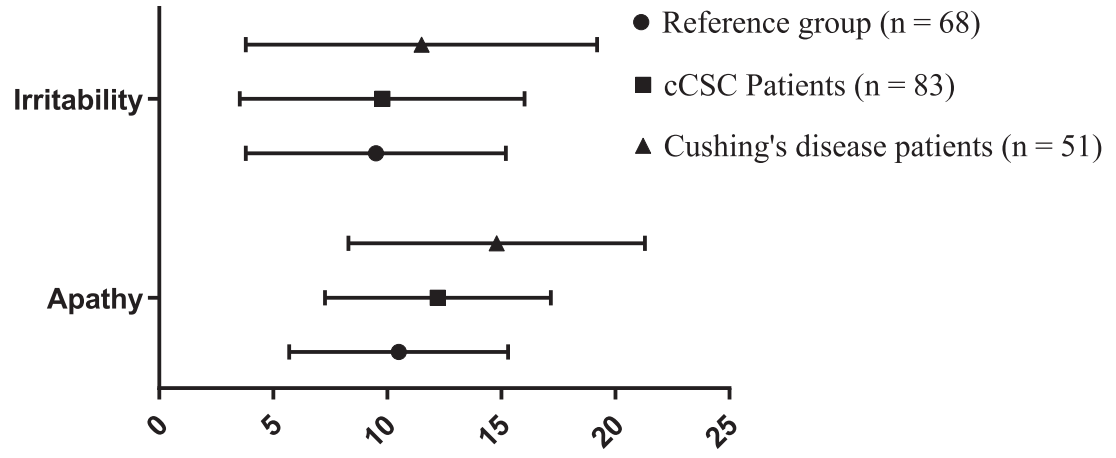

Fig. 2. Apathy and irritability in patients with cCSC. Data are presented as mean and SD. $\mathrm{cCSC}=$ chronic central serous chorioretinopathy; SD $=$ standard deviation.

Table 4. Coping in patients with cCSC.

\begin{tabular}{lllrlr}
\hline & $\begin{array}{l}\text { cCSC patients } \\
(n=83)\end{array}$ & $\begin{array}{l}\text { Reference data } \\
(n=1493)\end{array}$ & p-Value & $\begin{array}{l}\text { Cushing's disease } \\
\text { patients }(n=42)\end{array}$ & p-Value \\
\hline Active coping & $19.3(4.1)$ & $18.3(3.5)$ & 0.01 & $17.5(3.5)$ & 0.02 \\
Seeking distraction & $16.1(3.6)$ & $15.5(3.6)$ & 0.14 & $17.7(3.1)$ & 0.01 \\
Avoiding & $15.5(3.3)$ & $14.8(3.3)$ & 0.05 & $16.3(3.4)$ & 0.23 \\
Seeking social support & $12.6(2.7)$ & $11.3(3.0)$ & $<0.01$ & $13.3(4.0)$ & 0.28 \\
Passive coping & $11.8(3.3)$ & $10.7(2.9)$ & $<0.01$ & $12.0(3.4)$ & 0.71 \\
Expressing emotions & $5.7(1.6)$ & $6.2(1.7)$ & 0.01 & $5.9(1.6)$ & 0.65 \\
Fostering reassuring & $11.5(2.5)$ & $11.6(2.5)$ & 0.64 & $12.3(2.7)$ & 0.11 \\
thoughts & & & & & \\
\hline
\end{tabular}

Data are presented as mean (SD).

$\mathrm{cCSC}=$ chronic central serous chorioretinopathy; $\mathrm{SD}=$ standard deviation.

activated HPA axis in both patient groups, with patients with Cushing's disease at the far end of the spectrum of HPA - axis activation and patients with cCSC showing a slightly activated HPA axis, the present study showed there may also be similarity between patients with cCSC and patients treated for Cushing's disease regarding the spectrum of personality features. Despite this relative degree of similarity, there was no statistically significant difference in the tested personality traits between the current cCSC cohort and a healthy general population. In literature, a possible association between the occurrence of CSC and a combination of stressful life events and unfavourable coping styles has been reported, with patients with acute CSC reporting more unfavourable stress coping compared to patients with cCSC (Lahousen et al. 2016). Our current data suggest that patients with cCSC seek more social support. This may be explained by the fact that cCSC results in visual impairment affecting quality of life (Breukink et al. 2017), which makes patients more dependent on others. Moreover, patients with cCSC reported to use more active coping, but also more passive coping.
Although this may seem to be somewhat counterintuitive, it should be noted that coping behaviour is situation dependent, so that individuals can adapt their coping strategy based on the situation (Lazarus \& Folkman 1984).

The comparison of validated questionnaires outcome of a large cohort of patients with cCSC with both healthy controls and a cohort of patients treated for Cushing's disease enabled to describe personality traits, psychological morbidity and coping strategies within a broad spectrum of HPA - axis activity. Nevertheless, to find an ideal control group is challenging and the lack of a gender- and age-matched control group can be considered a potential limitation of this study. However, population-based reference data were available and considered to be a worthy alternative, since these data were derived from large populationbased cohorts (Dirk van Kampen 2009). The gender and age distribution of our cohort is in accordance with available literature (Liew et al. 2013; Daruich et al. 2015). Yet, since the majority of our population is male $(90 \%)$, our results may not be generalizable to female patients with cCSC.
This study aimed to investigate personality traits in patients with cCSC, and with the validated questionnaires used, we did not find an association with type A behavioural characteristics in these patients.

Using validated measures, we found no evidence for a higher prevalence of maladaptive personality traits such as type A behavioural characteristics in patients with cCSC, nor any clear differences in the generic personality traits as compared to the general population. This finding is of interest, as ophthalmologists often assume and report stress-related and type A behavioural characteristics in patients with cCSC, and therefore, the advice on stress reduction to these patients appears common in their management strategies (Gemenetzi et al. 2010; Rouvas et al. 2014; Goldhagen \& Goldhardt 2017). However, our paper indicates that psychological interventions targeting these personality features in cCSC, as was suggested in previous literature (Yannuzzi 1986; Conrad et al. 2014), may not be useful. The results of the present study contribute to the psychological phenotyping of patients with cCSC, which may be used to design disease-specific support programmes that address coping mechanisms in patients with cCSC.

\section{References}

Bahrke U, Krause A, Walliser U, Bandemer-Greulich U \& Goldhahn A (2000): Retinopathia centralis serosa-stomach ulcer of ophthalmology? Psychother Psychosom Med Psychol 50: $464-469$.

Baraki H, Feltgen N, Roider J, Hoerauf H \& Klatt C (2010): Central serous chorioretinopathy (CSC). Ophthalmologe 107: 479-492; quiz 493.

de Beurs E, Rinne T, van Kampen D, Verheul R \& Andrea H (2009): Reliability and validity of the Dutch Dimensional Assessment of Personality Pathology-Short Form (DAPP-SF), a shortened version of the DAPP-Basic Questionnaire. J Pers Disord 23: 308-326.

Bouzas EA, Karadimas P \& Pournaras CJ (2002): Central serous chorioretinopathy and glucocorticoids. Surv Ophthalmol 47: 431-448.

Brands I, Bol Y, Stapert S, Kohler S \& van Heugten C (2017): Is the effect of coping styles disease specific? Relationships with emotional distress and quality of life in acquired brain injury and multiple sclerosis Clin Rehabil 32: 116-126.

Breukink MB, Dingemans AJ, den Hollander AI et al. (2017): Chronic central serous chorioretinopathy: long-term follow-up and vision-related quality of life. Clin Ophthalmol (Auckland, N.Z.) 11: 39-46. Carvalho-Recchia CA, Yannuzzi LA, Negrao S, Spaide RF, Freund KB, Rodriguez-Coleman $\mathrm{H}$, Lenharo M \& Iida T (2002): Corticosteroids and central serous chorioretinopathy. Ophthalmology 109: 1834-1837.

Chatterjee A, Anderson KE, Moskowitz CB, Hauser WA \& Marder KS (2005): A comparison of self- 
report and caregiver assessment of depression, apathy, and irritability in Huntington's disease. J Neuropsychiatry Clin Neurosci 17: 378-383.

Chatziralli I, Kabanarou SA, Parikakis E, Chatzirallis A, Xirou T \& Mitropoulos P (2017): Risk factors for central serous chorioretinopathy: multivariate approach in a case-control study. Curr Eye Res 42: 1069-1073.

Conrad R, Bodeewes I, Schilling G, Geiser F, Imbierowicz K \& Liedtke R (2000): Central serous chorioretinopathy and psychological stress. Der Ophthalmologe: Zeitschrift der Deutschen Ophthalmologischen Gesellschaft 97: 527-531.

Conrad R, Geiser F, Kleiman A, Zur B \& KarpawitzGodt A (2014): Temperament and character personality profile and illness-related stress in central serous chorioretinopathy. ScientificWorldJournal 2014: 631687

Dalton SO, Boesen EH, Ross L, Schapiro IR \& Johansen C (2002): Mind and cancer. Do psychological factors cause cancer? Eur J Cancer 38: 1313-1323.

Daruich A, Matet A, Dirani A, Bousquet E, Zhao M, Farman N, Jaisser F \& Behar-Cohen F (2015): Central serous chorioretinopathy: recent findings and new physiopathology hypothesis. Prog Retin Eye Res 48: 82-118.

van Dijk EH, Dijkman G, Biermasz NR, van Haalen FM, Pereira AM \& Boon CJ (2016): Chronic central serous chorioretinopathy as a presenting symptom of Cushing syndrome. Eur J Ophthalmol 26: 442448.

Dirk van Kampen EB (2009): DAPP screening handleiding. The Netherlands: Hogrefe Uitgevers.

Donisan T, Bojinca VC, Dobrin MA et al. (2017): The relationship between disease activity, quality of life and personality types in rheumatoid arthritis and ankylosing spondylitis patients. Clin Rheumatol $\mathbf{3 6}$ 1511-1519.

Ersan N, Dolekoglu S, Fisekcioglu E, Ilguy M \& Oktay I (2017): Perceived sources and levels of stress, general self-efficacy and coping strategies in preclinical dental students. Psychol Health Med 22: $1175-1185$

Friedman M \& Rosenman RH (1959): Association of specific overt behavior pattern with blood and cardiovascular findings; blood cholesterol level, blood clotting time, incidence of arcus senilis, and clinical coronary artery disease. J Am Med Assoc 169: 1286-1296.

Friedman M, St George S, Byers SO \& Rosenman RH (1960): Excretion of catecholamines, 17-ketosteroids, 17-hydroxycorticoids and 5-hydroxyindole in men exhibiting a particular behavior pattern (A) associated with high incidence of clinical coronary artery disease. J Clin Invest 39: 758-764.

Friedman M, Byers SO, Diamant J \& Rosenman RH (1975): Plasma catecholamine response of coronaryprone subjects (type A) to a specific challenge. Metabolism 24: 205-210.

Friedman LC, Kalidas M, Elledge R, Chang J, Romero C, Husain I, Dulay MF \& Liscum KR (2006): Optimism, social support and psychosocial functioning among women with breast cancer. Psychooncology 15: 595-603.

Gemenetzi M, De Salvo G \& Lotery AJ (2010): Central serous chorioretinopathy: an update on pathogenesis and treatment. Eye (London, England) 24: 1743 1756.

Goldhagen BE \& Goldhardt R (2017): Diagnosed a patient with central serous chorioretinopathy? Now what?: management of central serous chorioretinopathy Curr Ophthalmol Rep 5: 141-148.

van Haalen FM, van Dijk EHC, Dekkers OM, Bizino MB, Dijkman G, Biermasz NR, Boon CJF \& Pereira AM (2018): Cushing's syndrome and hypothalamic-pituitary-adrenal axis hyperactivity in chronic central serous chorioretinopathy. Front Endocrinol (Lausanne) 9:39.

Hausteiner C, Klupsch D, Emeny R, Baumert J, Ladwig KH \& Investigators K (2010): Clustering of negative affectivity and social inhibition in the community: prevalence of type $\mathrm{D}$ personality as a cardiovascular risk marker. Psychosom Med 72: 163-171.

Hopman-Rock M, Kraaimaat FW \& Bijlsma JW (1997): Quality of life in elderly subjects with pain in the hip or knee. Qual Life Res 6: 67-76.

Jenkins CD, Rosenman RH \& Friedman M (1967): Development of an objective psychological test for the determination of the coronary-prone behavior pattern in employed men. J Chronic Dis 20: $371-379$.

Jonas JB \& Kamppeter BA (2005): Intravitreal triamcinolone acetonide and central serous chorioretinopathy. Br J Ophthalmol 89: 386-387.

van Kampen D, de Beurs E \& Andrea H (2008): A short form of the dimensional assessment of personality pathology-basic questionnaire (DAPP-BQ): the DAPP-SF. Psychiatry Res 160: 115-128.

Keramat Kar M, Whitehead L \& Smith CM (2017): Characteristics and correlates of coping with multiple sclerosis: a systematic review. Disabil Rehabil 10: $1-15$.

Lahousen T, Painold A, Luxenberger W, Schienle A, Kapfhammer HP \& Ille R (2016): Psychological factors associated with acute and chronic central serous chorioretinopathy. Nord J Psychiatry 70: 24-30.

Lazarus RS \& Folkman S (1984): Stress, appraisal and coping. New York: Springer.

Liew G, Quin G, Gillies M \& Fraser-Bell S (2013): Central serous chorioretinopathy: a review of epidemiology and pathophysiology. Clin Exp Ophthalmol 41: 201-214.

Liu B, Deng T \& Zhang J (2016): Risk Factors for Central Serous Chorioretinopathy: a systematic review and meta-analysis. Retina 36: 9-19.

Nicholson B, Noble J, Forooghian F \& Meyerle C (2013): Central serous chorioretinopathy: update on pathophysiology and treatment. Surv Ophthalmol 58: $103-126$.

Rosenman RH, Brand RJ, Sholtz RI \& Friedman M (1976): Multivariate prediction of coronary heart disease during 8.5 year follow-up in the Western Collaborative Group Study. Am J Cardiol 37: 903-910.

Rouvas AA, Chatziralli IP, Ladas ID et al. (2014): The impact of financial crisis on central serous chorioretinopathy in Greece: is there any correlation? Eur J Ophthalmol 24: 559-565.

Schouws SN, Paans NP, Comijs HC, Dols A \& Stek ML (2015): Coping and personality in older patients with bipolar disorder. J Affect Disord 184: 67-71.

Schreurs PJG, Tellegen B \& Van de Willige G (1984): Gezondheid, stress en coping: de ontwikkeling van de Utrechtse Coping Lijst. Gedrag: tijdschrift voor psychologie 12: 101-117.

Schreurs PJG, van de Willige G, Brosschot JF, Tellegen B \& Graus GHM (1993): De Utrechtse coping lijst: UCL. Lisse: Swets en Zeitlinger.

Spahn C, Wiek J \& Burger T (2004): Operationalized psychodynamic diagnostics (OPD) in patients with central serous chorioretinopathy. Psychother Psychosom Med Psychol 54: 52-57.

Starkstein SE, Petracca G, Chemerinski E \& Kremer J (2001): Syndromic validity of apathy in Alzheimer's disease. Am J Psychiatry 158: 872-877.

Tiemensma J, Biermasz NR, Middelkoop HA, van der Mast RC, Romijn JA \& Pereira AM (2010a): Increased prevalence of psychopathology and maladaptive personality traits after long-term cure of Cushing's disease. J Clin Endocrinol Metab 95: E129-E141.

Tiemensma J, Biermasz NR, van der Mast RC, Wassenaar MJ, Middelkoop HA, Pereira AM \& Romijn JA (2010b): Increased psychopathology and maladaptive personality traits, but normal cognitive functioning, in patients after long-term cure of acromegaly. J Clin Endocrinol Metab 95: E392-E402.

Tiemensma J, Kaptein AA, Pereira AM, Smit JW, Romijn JA \& Biermasz NR (2011): Coping strategies in patients after treatment for functioning or nonfunctioning pituitary adenomas. J Clin Endocrinol Metabol 96: 964-971.
Vollmann M, Pukrop J \& Salewski C (2016): Coping mediates the influence of personality on life satisfaction in patients with rheumatic diseases. Clin Rheumatol 35: 1093-1097.

Wadden TA, Anderton CH, Foster GD \& Love W (1983): The Jenkins activity survey: does it measure psychopathology? J Psychosom Res 27: 321-325.

Wang M, Munch IC, Hasler PW, Prunte C \& Larsen M (2008): Central serous chorioretinopathy. Acta Ophthalmol 86: 126-145.

Williams RB Jr, Lane JD, Kuhn CM, Melosh W, White AD \& Schanberg SM (1982): Type A behavior and elevated physiological and neuroendocrine responses to cognitive tasks. Science 218: 483-485.

Yadav P, Bhattacharyya D, Srivastava K \& Salhotra N (2017): Study of personality traits, individual coping resources, and their association in HIV-seropositive males. Ind Psychiatry J 26: 45-51

Yannuzzi LA (1986): Type A behavior and central serous chorioretinopathy. Trans Am Ophthalmol Soc 84: 799-845.

Yannuzzi LA (1987): Type-A behavior and central serous chorioretinopathy. Retina (Philadelphia, Pa.) 7: 111-131.

Yannuzzi LA (2010): Central serous chorioretinopathy: a personal perspective. Am J Ophthalmol 149: 361-363.

You J, Wang C, Rodriguez L, Wang X \& Lu Q (2018): Personality, coping strategies and emotional adjustment among Chinese cancer patients of different ages. Eur J Cancer Care (Engl) 1.

Received on June 28th, 2018.

Accepted on September 26th, 2018.

\section{Correspondence}

Camiel J.F. Boon, MD, PhD, FEBOphth

Department of Ophthalmology

Leiden University Medical Center

P.O. Box 9600

2300 RC Leiden

the Netherlands

Tel: $+31-71-5265936$

Fax: + 31-71-5248222

Email: c.j.f.boon@1umc.nl

${ }^{\dagger}$ These authors contributed equally to this paper.

The authors would like to thank M.B. Bizino, D Mohabati and I.C.M. Pelsma for their help conducting this research.

This research was supported by the following foundations: MaculaFonds, Retina Netherlands, Blinden-Penning and Landelijke Stichting voor Blinden en Slechtzienden, which contributed through UitZicht, as well as Rotterdamse Stichting Blindenbelangen, Stichting Blindenhulp, ZonMw VENI Grant (CJFB) and Gisela Thier Fellowship of Leiden University (CJFB). The funding organizations had no role in the design or conduct of this research. They provided unrestricted grants.

\section{Supporting Information}

Additional Supporting Information may be found in the online version of this article:

Appendix S1. Post-hoc analysis without patients with less typical cCSC. 